\title{
Letter
}

\section{Effective transverse radius of nucleon in high-energy elastic diffractive scattering}

\author{
A. A. Godizov ${ }^{\text {a }}$ \\ Institute for High Energy Physics, NRC “Kurchatov Institute”, 142281 Protvino, Russia
}

Received: 13 April 2015 / Accepted: 9 May 2015 / Published online: 22 May 2015

(C) The Author(s) 2015. This article is published with open access at Springerlink.com

\begin{abstract}
High-energy elastic diffraction of nucleons is considered in the framework of the simplest Regge-eikonal approximation. It is demonstrated explicitly that the effective transverse radius of nucleon in this nonperturbative regime is $\sim 0.2 \div 0.3 \mathrm{fm}$ and much less than the transverse size of the diffractive interaction region.
\end{abstract}

\section{Introduction}

Elastic diffractive scattering of hadrons is one of the most interesting and important areas of high-energy hadron physics: in $p p$ collisions the fraction of elastic diffraction events in the total number of events is very high (from more than $15 \%$ at the ISR to about $25 \%$ at the LHC). However, the main problem related to this sector of strong interaction physics is the fact that the characteristic distances for diffractive interaction of hadrons are of order $1 \mathrm{fm}$, so that perturbative QCD is inapplicable. Hence, one has to search for some approaches not related to $\mathrm{pQCD}$ directly, to provide an at least qualitative description of the corresponding high-energy observables.

One of the most natural theoretical frameworks which helps to deal with the nonperturbative sector of hadron physics is Regge theory, wherein the interaction of hadrons is described in terms of exchanges by reggeons (off-mass-shell and off-spin-shell composite particles). In Ref. [1] a simple Regge-eikonal model for high-energy elastic diffraction of nucleons was examined. In this model, the standard eikonal representation of the nonflip scattering amplitude [2],

$$
\begin{gathered}
T_{e l}(s, t)=4 \pi s \int_{0}^{\infty} \mathrm{d} b^{2} J_{0}(b \sqrt{-t}) \frac{e^{2 i \delta(s, b)}-1}{2 i}, \\
\delta(s, b)=\frac{1}{16 \pi s} \int_{0}^{\infty} \mathrm{d}(-t) J_{0}(b \sqrt{-t}) \delta(s, t)
\end{gathered}
$$

\footnotetext{
a e-mail: anton.godizov@gmail.com
}

(here $s$ and $t$ are the Mandelstam variables and $b$ is the impact parameter), is exploited together with the single-reggeonexchange approximation to the eikonal (Born amplitude) in the kinematic range $s \gg\left\{m_{p}^{2},|t|\right\}$ :

$\delta(s, t)=\delta_{\mathrm{P}}(s, t)=\left(i+\operatorname{tg} \frac{\pi\left(\alpha_{\mathrm{P}}(t)-1\right)}{2}\right) \Gamma_{\mathrm{P}}^{2}(t)\left(\frac{s}{s_{0}}\right)^{\alpha_{\mathrm{P}}(t)}$,

where $s_{0}=1 \mathrm{GeV}^{2}$ and $\alpha_{\mathrm{P}}(t)$ is the Regge trajectory of a pomeron (a $C$-even reggeon, which absolutely dominates over other reggeons at the SPS, Tevatron, and LHC energies). More details, regarding the Regge-eikonal approach, can be found in [2] or, partly, in the appendix.

In [1] the unknown functions $\alpha_{\mathrm{P}}(t)$ and $\Gamma_{\mathrm{P}}(t)$ are considered independent and treated with the help of some simple test parametrizations which are fitted to the data. Formally, the used parametrizations have provided a satisfactory description of the available experimental data at $\sqrt{s}>500 \mathrm{GeV}$ and $-t<2 \mathrm{GeV}^{2}$. However, a question emerges about possible correlation between the behavior of $\alpha_{\mathrm{P}}(t)$ and $\Gamma_{\mathrm{P}}(t)$ at low $t$, since both functions have a strong impact on the $t$-behavior of the scattering amplitude.

Such a correlation exists and can be taken into account explicitly. As a consequence, it becomes possible to extract from the data valuable information concerning the effective transverse radius of nucleon in the nonperturbative regime of high-energy diffractive scattering.

\section{Structure of the pomeron Regge residue}

First of all, we should note that in the framework of the Regge-eikonal approach the pomeron exchange contribution into the eikonal of nucleon-nucleon elastic scattering appears as 
$\delta_{\mathrm{P}}(s, t)=\left(i+\operatorname{tg} \frac{\pi\left(\alpha_{\mathrm{P}}(t)-1\right)}{2}\right) g_{\mathrm{P}}^{2}(t) \pi \alpha_{\mathrm{P}}^{\prime}(t)\left(\frac{s}{2 s_{0}}\right)^{\alpha_{\mathrm{P}}(t)}$,

where $\alpha_{\mathrm{P}}^{\prime}(t)$ originates from the pomeron propagator (see the appendix) and, in general, the factor $2^{-\alpha_{\mathrm{P}}(t)} \pi \alpha_{\mathrm{P}}^{\prime}(t)$ is not related to the pomeron-nucleon coupling. In the literature this factor is usually included into the corresponding Regge residue [2]: $g_{\mathrm{P}}^{2}(t) 2^{-\alpha_{\mathrm{P}}(t)} \pi \alpha_{\mathrm{P}}^{\prime}(t) \equiv \Gamma_{\mathrm{P}}^{2}(t)$. The real part of the eikonal has simple poles at those values of $t$ where the pomeron Regge trajectory takes on even integers: $\alpha_{\mathrm{P}}\left(M_{j}^{2}-\right.$ $\left.i M_{j} \Gamma_{j}\right)=j=2 n$ (here $j, M_{j}$, and $\Gamma_{j}$ are the glueball spin, mass, and decay width). The existence of such poles provides the corresponding glueball resonance contributions into the $t$-channel amplitude.

If to consider $g_{\mathrm{P}}(t)$ as a nontrivial unknown function of $t$, then expressions (2) and (3) are equivalent from the standpoint of description of data. However, the replacement $g_{\mathrm{P}}(t) \rightarrow \Gamma_{\mathrm{P}}(t)$ degrades the physical transparency of the model, since the shortened form (2) ignores the evident correlation between the behavior of the pomeron Regge residue and the pomeron Regge trajectory. Moreover, if the $t$-dependence of $g_{\mathrm{P}}(t)$ is weak at low $t$, then usage of (2) instead of (3) leads to the loss of physical information.

Treating the high-energy elastic scattering of nucleons in the same way as the lepton-proton elastic scattering, we define the quantity $g_{\mathrm{P}}(0)$ as the effective "pomeron charge" of nucleon, while the ratio $g_{\mathrm{P}}(t) / g_{\mathrm{P}}(0)$ should be considered as the nucleon "pomeron form factor". By analogy with the extraction of the proton charge radius from the proton charge form factor, one can extract the effective transverse "pomeron radius" of the nucleon from $g_{\mathrm{P}}(t)$. Hence, a possible weak $t$-dependence of this quantity at low $t$ could be interpreted as the effective transverse (quasi-)pointlikeness of the nucleon in the high-energy diffractive scattering regime.

Let us recall that although QCD itself does not predict the behavior of $\alpha_{\mathrm{P}}(t)$ in the diffraction domain $(0<-t<$ $2 \mathrm{GeV}^{2}$ ), this function is expected to satisfy the following conditions $[2,3]$ :

$$
\frac{\mathrm{d}^{n} \alpha_{\mathrm{P}}}{\mathrm{d} t^{n}}>0 \quad(n=1,2, \ldots ; t<0), \quad \lim _{t \rightarrow-\infty} \alpha_{\mathrm{P}}(t)=1
$$

The first condition originates from the dispersion relations for Regge trajectories (if not more than one subtraction is needed), and the second one follows from the natural presumption that at high values of the transferred momentum the exchange by a pomeron turns, due to asymptotic freedom, into the exchange by two noninteracting gluons, which can be considered in the same way as the exchange by two photons. At high energies such Born amplitudes behave as $\sim s^{1}[4]$.
Thus, if the effective transverse radius of nucleon is small, then, in view of restrictions (4), the $t$-dependence of eikonal (3) is determined mainly by the Herglotz function $\alpha_{\mathrm{P}}(t)$. Consequently, the quality of the description of the differential cross section $\frac{\mathrm{d} \sigma}{\mathrm{d} t}=\frac{|T(s, t)|^{2}}{16 \pi s^{2}}$ becomes extremely sensitive to the quantitative behavior of $\alpha_{\mathrm{P}}(t)$ at low negative $t$. Such a sensitivity implies that the procedure of fitting $\alpha_{\mathrm{P}}(t)$ and $g_{\mathrm{P}}(0)$ to experimental angular distributions in a wide enough kinematic range could be considered as an implicit extraction of these quantities from the data.

\section{Fitting to the experimental data}

Let us reconsider the model from [1], having singled out the factor $2^{-\alpha_{\mathrm{P}}(t)} \pi \alpha_{\mathrm{P}}^{\prime}(t)$ in the Regge residue, as in (3):

$\alpha_{\mathrm{P}}(t)=1+\frac{\alpha_{\mathrm{P}}(0)-1}{1-\frac{t}{\tau_{a}}}, \quad \Gamma_{\mathrm{P}}(t) \rightarrow g_{\mathrm{P}}(t)=\frac{g_{\mathrm{P}}(0)}{\left(1-a_{g} t\right)^{2}}$

The results of fitting $\alpha_{\mathrm{P}}(t)$ and $g_{\mathrm{P}}(t)$ to the experimental differential cross sections at $\sqrt{s}>500 \mathrm{GeV}$ and 0.005 $\mathrm{GeV}^{2}<-t<2 \mathrm{GeV}^{2}$ [5-14] are presented in Tables 1, 2, and 3 and Fig. 1. The deviation of the model predictions from the $p p$ elastic scattering data in the dip region at $\sqrt{s}=62.5 \mathrm{GeV}$ [15] can be explained by the noticeable contribution of secondary reggeons into the real part of the eikonal. A detailed discussion of this matter can be found in [1].

The D0 data [16] were not included into the fitting procedure, since they have a normalization uncertainty about

Table 1 The parameter values obtained via fitting to the high-energy differential cross-section data

\begin{tabular}{ll}
\hline Parameter & Value \\
\hline$\alpha_{\mathrm{P}}(0)-1$ & $0.109 \pm 0.017$ \\
$\tau_{a}$ & $(0.535 \pm 0.057) \mathrm{GeV}^{2}$ \\
$g_{\mathrm{P}}(0)$ & $(13.8 \pm 2.3) \mathrm{GeV}$ \\
$a_{g}$ & $(0.23 \pm 0.07) \mathrm{GeV}^{-2}$ \\
\hline
\end{tabular}

Table 2 The quality of description of the data [5-14] on the angular distributions of nucleon-nucleon scattering

\begin{tabular}{lrr}
\hline$\sqrt{s}(\mathrm{GeV})$ & Number of points & $\chi^{2}$ \\
\hline $546(\bar{p} p ; \mathrm{UA} 1, \mathrm{UA} 4, \mathrm{CDF})$ & 231 & 253 \\
$630(\bar{p} p ; \mathrm{UA} 4)$ & 17 & 11 \\
$1800(\bar{p} p ; \mathrm{E} 710)$ & 51 & 16 \\
$7000(p p ;$ TOTEM, ATLAS $)$ & 201 & 188 \\
Total & 500 & 468 \\
\hline
\end{tabular}


$14.4 \%$. If to multiply them by a factor 0.92 , the quality of the description becomes much better (see Table 4). The same can be said regarding the unrenormalized CDF data at $\sqrt{s}=$ $1800 \mathrm{GeV}$ [17], which are inconsistent with the E-710 data.

Table 3 Predictions for the $p p$ total and elastic cross sections and the forward logarithmic slope of the corresponding differential crosssections

\begin{tabular}{lcrl}
\hline$\sqrt{s}(\mathrm{GeV})$ & $\sigma_{\text {tot }}(\mathrm{mb})$ & \multicolumn{1}{c}{$\sigma_{\mathrm{el}}(\mathrm{mb})$} & $B\left(\mathrm{GeV}^{-2}\right)$ \\
\hline 62.5 & $43.0 \pm 4.4$ & $7.4 \pm 1.2$ & $14.5 \pm 0.8$ \\
200 & $53.3 \pm 3.8$ & $10.2 \pm 1.2$ & $16.0 \pm 0.8$ \\
546 & $63.8 \pm 3.3$ & $13.2 \pm 1.0$ & $17.3 \pm 0.9$ \\
1800 & $78.5 \pm 3.4$ & $17.8 \pm 1.0$ & $19.1 \pm 1.0$ \\
7000 & $98.4 \pm 5.4$ & $24.5 \pm 1.8$ & $21.4 \pm 1.1$ \\
8000 & $100.5 \pm 5.7$ & $25.3 \pm 1.9$ & $21.6 \pm 1.1$ \\
13000 & $108.6 \pm 6.9$ & $28.2 \pm 2.5$ & $22.5 \pm 1.2$ \\
14000 & $109.9 \pm 7.1$ & $28.6 \pm 2.5$ & $22.6 \pm 1.2$ \\
32000 & $124.9 \pm 9.7$ & $34.1 \pm 3.7$ & $24.2 \pm 1.4$ \\
100000 & $148.0 \pm 14.1$ & $42.8 \pm 5.6$ & $26.6 \pm 1.7$ \\
\hline
\end{tabular}
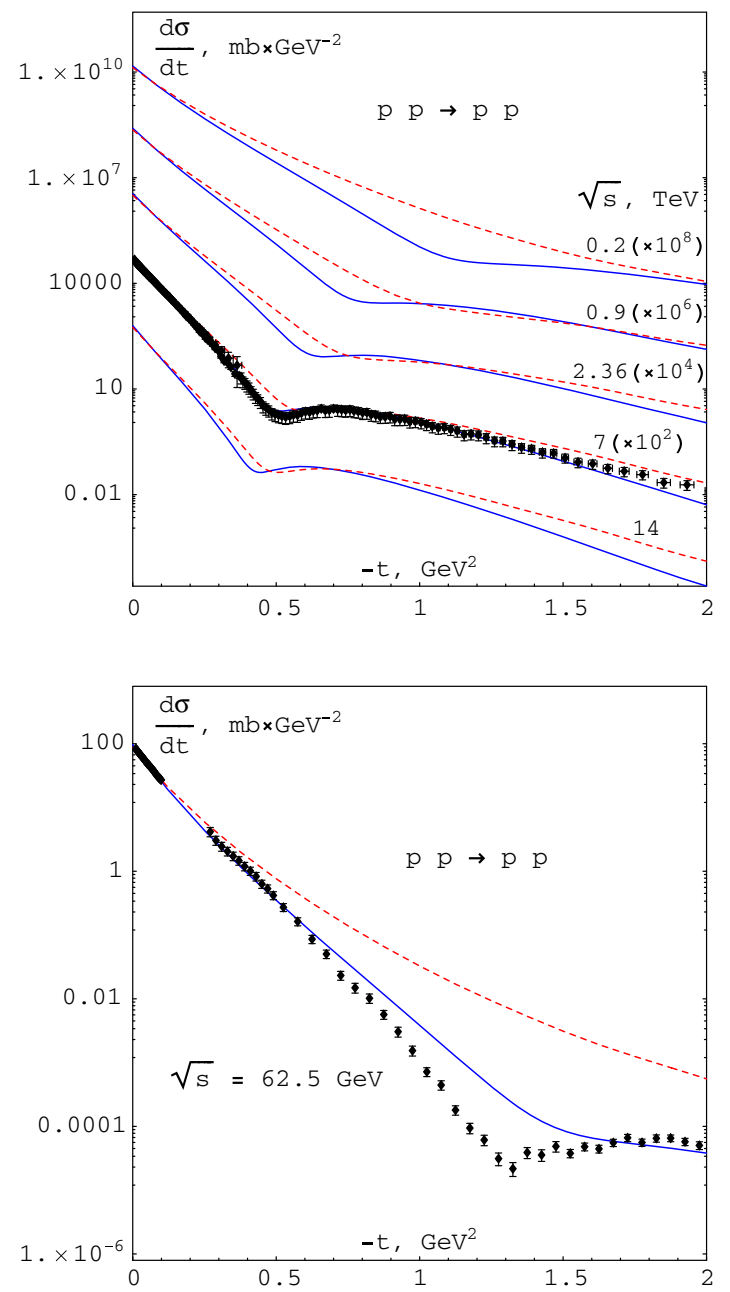

\section{Justification for usage of the parametrization for $\alpha_{\mathrm{P}}(t)$}

Before drawing any physical conclusions from the fitting results, we should discuss our choice of parametrization for $\alpha_{\mathrm{P}}(t)$. First, the essential nonlinearity of $\alpha_{\mathrm{P}}(t)$ from (5), which, in fact, is not related to this concrete expression but follows from the restrictions (4), seems to be in contradiction with the observed approximate linearity of the ChewFrautschi plots for secondary reggeons. However, we would like to point out that such a linear behavior of secondaries takes place at $t>0$ and, in principle, is not guaranteed for $t<0$. Moreover, it was determined in the framework of the BFKL approach [18,19] that secondary Regge trajectories behave as $\alpha_{\mathrm{R}}(t)=\left(\frac{8 \alpha_{s}(\sqrt{-t})}{3 \pi}\right)^{1 / 2}+O\left(\alpha_{s}^{5 / 6}(\sqrt{-t})\right)$ at high negative $t$, where $\alpha_{s}(\mu)$ is the QCD running coupling. Hence, in view of the rather high slopes of the corresponding Chew-Frautschi plots in the resonance region, we have a very simple alternative: either secondary Regge trajectories are essentially nonlinear in the diffraction domain, or they
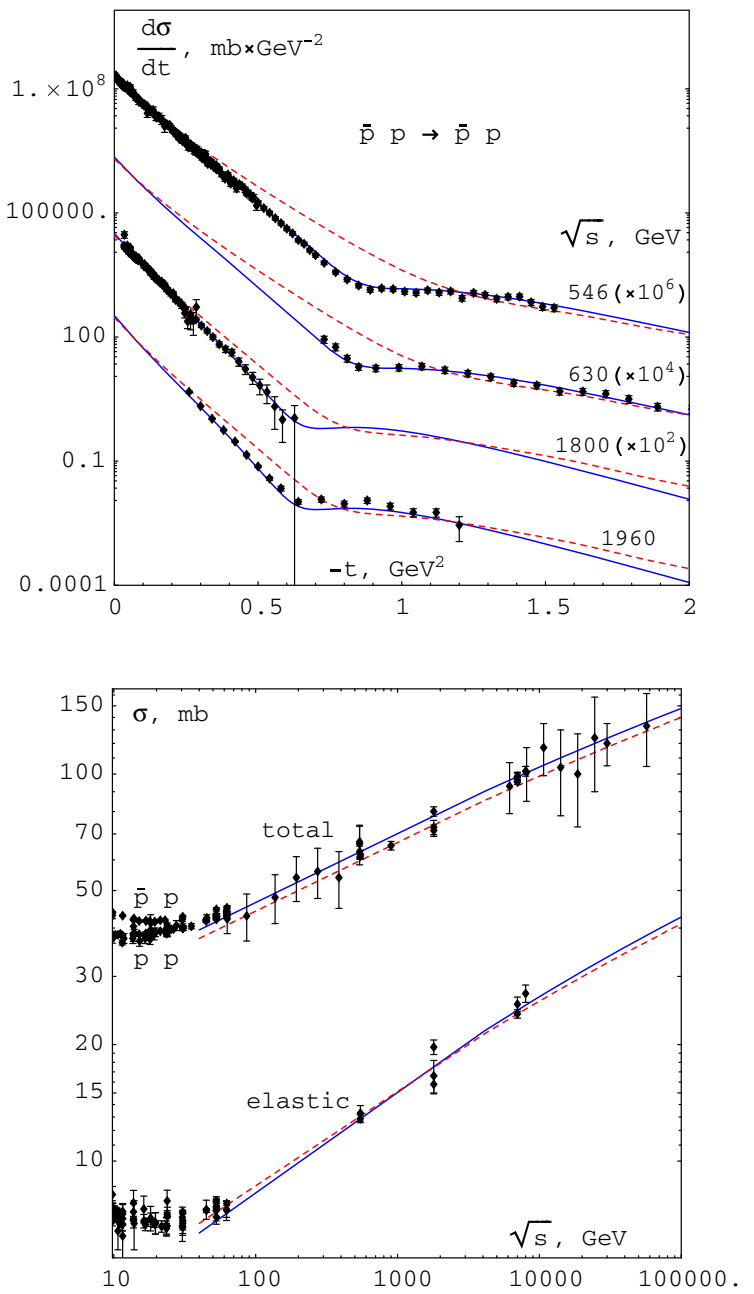

Fig. 1 The high-energy evolution of the nucleon-nucleon elastic scattering observables. The dashed lines correspond to the value $a_{g}=0$ instead of $a_{g}=0.23 \mathrm{GeV}^{-2}$ 
Table 4 The quality of description of the data $[16,17]$ not included into the fitting procedure

\begin{tabular}{llr}
\hline$\sqrt{s}(\mathrm{GeV})$ & Number of points & $\chi^{2}$ \\
\hline $1960(\bar{p} p ; \mathrm{D} 0)$ & 17 & 55 \\
$1960(\bar{p} p ; \mathrm{D} 0$, multiplied by 0.92$)$ & 17 & 29 \\
$1800(\bar{p} p ; \mathrm{CDF})$ & 26 & 178 \\
$1800(\bar{p} p ; \mathrm{CDF}$, multiplied by 0.88$)$ & 26 & 45 \\
\hline
\end{tabular}

are not monotonic functions in the interval $-\infty<t<0$. A detailed discussion of this matter can be found in [20].

Second, we should ascertain that the obtained smallness of $a_{g}$ (see Table 1) is not related directly to the chosen specific parametrization for $\alpha_{\mathrm{P}}(t)$, since this is crucial for the main conclusion of the paper. If we consider a simple generalization of (5),

$\alpha_{\mathrm{P}}^{(k)}(t)=1+\frac{\alpha_{\mathrm{P}}^{(k)}(0)-1}{\left(1-\frac{t}{\tau_{a}^{(k)}}\right)^{k}}, \quad g_{\mathrm{P}}^{(k)}(t)=\frac{g_{\mathrm{P}}^{(k)}(0)}{\left(1-a_{g}^{(k)} t\right)^{2}}$,

where $k$ takes on integer and half-integer values [the parametrization (5) corresponds to $k=1$ ], then it is possible to provide a satisfactory description of the data in a few cases of this series (see Table 5). The description for $k=1 / 2$ and $k \geq 3$ is unsatisfactory. For any $k>1$, we find that $a_{g}^{(k)}<a_{g}^{(1)}$. Thus, the conclusion we draw below is not related to the specific form (5) of $\alpha_{\mathrm{P}}(t)$ only.

At the very end of this section we would like to note that although in this paper we restricted ourselves to the simplest test parametrizations, the quantities $\alpha_{\mathrm{P}}(t)$ and $g_{\mathrm{P}}(t)$ should, in general, be treated as unknown functions in the framework of the considered model. Namely, any expression for $\alpha_{\mathrm{P}}(t)$ could be used which is analytic at $t<0$ and satisfies the conditions (4). Certainly, it should provide a satisfactory description of the available data as well.

\section{Discussion}

Now let us analyze the outcomes produced.

The description of the nucleon-nucleon diffractive pattern in the considered kinematic range is satisfactory. The replacement of the fitted value of $a_{g}$ by $a_{g}=0$, which implies the neglect of the nucleon shape, disfigures the differential cross sections (see the dashed lines in Fig. 1). However, this distortion decreases with energy and does not become catastrophic already at the LHC energies. It is a consequence of the fact that, although we did not fix the nucleon transverse radius, it has turned out rather small. Indeed, the form factor $g_{\mathrm{P}}(t) / g_{\mathrm{P}}(0)=\left(1-a_{g} t\right)^{-2}$ corresponds to a certain effective transverse distribution in the impact parameter representation: $f(b)=\left(4 \pi a_{g}^{3}\right)^{-1} b K_{1}\left(b / a_{g}\right)$, where $K_{1}(x)$ is the modified Bessel function. The effective transverse radius of the nucleon obtained via an average over this distribution, $\sqrt{\left\langle b^{2}\right\rangle} \sim 0.2 \div 0.3 \mathrm{fm}$, is noticeably smaller than the effective transverse size of the diffractive interaction region (in the considered interval of the collision energy $\sqrt{2 B}>1 \mathrm{fm}$, $B$ is the forward logarithmic slope of $\mathrm{d} \sigma / \mathrm{d} t$, which increases with energy). Such a difference could be interpreted as if the pomeron were coupled to a very small zone inside nucleon. Hence, we arrive at the main conclusion:

- The quantitative evolution of the nucleon-nucleon elastic diffractive scattering observables at ultrahigh energies is determined mainly by the behavior of the pomeron Regge trajectory $\alpha_{\mathrm{P}}(t)$ and the value of the effective "pomeron charge" $g_{\mathrm{P}}(0)$ of nucleon and rather weakly depends on the nucleon shape.

The examined single-reggeon-exchange eikonal approximation is expected to be valid at least in the interval 0.2 $\mathrm{TeV} \leq \sqrt{s} \leq 14 \mathrm{TeV}$. Therefore, the forthcoming TOTEM measurements (as well as desirable analogous measurements at the RHIC) could decrease the uncertainties of the model degrees of freedom and, thus, improve the phenomenological estimations as for the nucleon effective transverse radius, and therefore for the pomeron Regge trajectory and the pomeron charge of nucleon.

Acknowledgments The author thanks V. A. Petrov and R. A. Ryutin for discussion.

Open Access This article is distributed under the terms of the Creative Commons Attribution 4.0 International License (http://creativecomm ons.org/licenses/by/4.0/), which permits unrestricted use, distribution, and reproduction in any medium, provided you give appropriate credit
Table 5 The fitting results for different variants of parametrization (6)

\begin{tabular}{llll}
\hline Parameter & $k=3 / 2$ & $k=2$ & $k=5 / 2$ \\
\hline$\alpha_{\mathrm{P}}^{(k)}(0)-1$ & $0.108 \pm 0.017$ & $0.108 \pm 0.017$ & $0.108 \pm 0.017$ \\
$\tau_{a}^{(k)}$ & $(0.708 \pm 0.079) \mathrm{GeV}^{2}$ & $(0.874 \pm 0.100) \mathrm{GeV}^{2}$ & $(1.040 \pm 0.125) \mathrm{GeV}^{2}$ \\
$g_{\mathrm{P}}^{(k)}(0)$ & $(13.1 \pm 2.2) \mathrm{GeV}$ & $(12.6 \pm 2.0) \mathrm{GeV}$ & $(12.3 \pm 2.0) \mathrm{GeV}$ \\
$a_{g}^{(k)}$ & $(0.167 \pm 0.075) \mathrm{GeV}^{-2}$ & $(0.120 \pm 0.077) \mathrm{GeV}^{-2}$ & $(0.085 \pm 0.080) \mathrm{GeV}^{-2}$ \\
$\chi^{2} / \mathrm{DoF}$ & 0.915 & 0.93 & 0.97 \\
\hline
\end{tabular}


to the original author(s) and the source, provide a link to the Creative Commons license, and indicate if changes were made. Funded by SCOAP ${ }^{3}$.

\section{Appendix. Regge approximation for Born amplitude}

The eikonal representation (1) itself implies just a replacement of the unknown function of two variables, $T(s, t)$, by another one, $\delta(s, t)$. The key assumption is that the eikonal is proportional (with high accuracy) to some effective relativistic quasi-potential of two-hadron interaction. According to the Van Hove interpretation of such a quasi-potential as the "sum" over all single-meson exchanges in the $t$-channel [21], the eikonal can be represented as

$$
\begin{aligned}
\delta= & \sum_{j=0}^{\infty} \sum_{m_{j}} J_{\alpha_{1}, \ldots, \alpha_{j}}^{\left(f_{1}, j, m_{j}\right)}\left(p_{1}, \Delta\right) \\
& \times \frac{D_{\left(j, m_{j}\right)}^{\alpha_{1}, \ldots, \alpha_{j}, \beta_{1}, \ldots, \beta_{j}}(\Delta)}{m_{j}^{2}-\Delta^{2}} J_{\beta_{1}, \ldots, \beta_{j}}^{\left(f_{2}, j, m_{j}\right)}\left(p_{2}, \Delta\right),
\end{aligned}
$$

where $\frac{D_{\left(j, m_{j}\right)}^{\alpha_{1}, \ldots, \alpha_{j}, \beta_{1}, \ldots, \beta_{j}}}{m_{j}^{2}-\Delta^{2}}$ is the propagator of spin- $j$ meson particle, $m_{j}^{2}=M_{j}^{2}-i M_{j} \Gamma_{j}\left(M_{j}\right.$ and $\Gamma_{j}$ are the meson mass and decay width), $J_{\alpha_{1}, \ldots, \alpha_{j}}^{\left(f, j, m_{j}\right)}$ are the corresponding meson currents of interacting hadrons (index $f$ denotes the sort of the hadron), $\Delta$ is the transferred 4-momentum, $p_{1}$ and $p_{2}$ are the 4-momenta of the incoming particles, and symbol $\sum_{m_{j}}$ denotes the summing over all of spin- $j$ mesons with different masses (which, in what further, will be transformed into the summing over reggeons).

Obviously, in the kinematic range $\left(p_{1}+p_{2}\right)^{2} \equiv s \gg$ $\left\{p_{1,2}^{2}, \Delta^{2},\left(p_{1,2} \Delta\right)\right\}$ the eikonal can be approximated by the following expression:

$\delta(s, t)=\sum_{j=0}^{\infty} \sum_{m_{j}} \frac{h^{\left(j, m_{j}\right)}(t)}{m_{j}^{2}-t}\left(\frac{s}{2}\right)^{j}$,

where $t \equiv \Delta^{2}, h^{\left(j, m_{j}\right)}(t) \equiv F^{\left(f_{1}, j, m_{j}\right)}\left(p_{1}^{2}, \Delta^{2}\right.$, $\left.\left(p_{1} \Delta\right)\right) F^{\left(f_{2}, j, m_{j}\right)}\left(p_{2}^{2}, \Delta^{2},\left(p_{2} \Delta\right)\right)$, and $F^{\left(f, j, m_{j}\right)}$ is the structure function at the tensor structure $p_{\alpha_{1}}, \ldots, p_{\alpha_{j}}$ in the current $J_{\alpha_{1}, \ldots, \alpha_{j}}^{\left(f, j, m_{j}\right)}(p, \Delta)$. Certainly, the symmetric tensors $J_{\alpha_{1}, \ldots, \alpha_{j}}^{\left(f, j, m_{j}\right)}$ should be transverse with respect to $\Delta^{\alpha_{k}}$ and traceless, for the unambiguous interpretation of $j$ as the massive meson spin, though these transversality and tracelessness are not used in the leading approximation.

Now let us introduce the single-meson-exchange amplitudes of definite signature:

$\delta_{ \pm}(s, t)=\sum_{j=0}^{\infty} \sum_{m_{j}}\left(1 \pm e^{-i \pi j}\right) \frac{h^{\left(j, m_{j}\right)}(t)}{m_{j}^{2}-t}\left(\frac{s}{2}\right)^{j}$.
If $m_{j}^{2}$ and $h^{\left(j, m_{j}\right)}(t)$ at even and odd $j$ are the values of some analytic functions which are holomorphic at $\operatorname{Re} j>-\frac{1}{2}$ and behave as $O\left(e^{k|j|}\right), k<\pi$, at $j \rightarrow \infty$, then, under the Carlson theorem [22], the unilocal analytic continuation of (A.3) into the region of complex $j$ is possible (the Regge hypothesis [2]). We denote these functions by $m_{ \pm}^{2}(j)$ and $h_{ \pm}\left(t, j, m_{ \pm}^{2}(j)\right)$, respectively. Via the Sommerfeld-Watson transform $[2,23,24]$, we replace the sum over $j$ in (A.3) by the integral over the contour $C$ encircling the real positive half-axis on the complex $j$-plane, including the point $j=0$, in such a way that the half-axis is on the right:

$$
\begin{aligned}
\delta_{ \pm}(s, t)= & \frac{1}{2 i} \oint_{C} \frac{\mathrm{d} j}{\sin (\pi j)} \sum_{m_{ \pm}}\left(\mp 1-e^{-i \pi j}\right) \\
& \times \frac{h_{ \pm}\left(t, j, m_{ \pm}^{2}(j)\right)}{m_{ \pm}^{2}(j)-t}\left(\frac{s}{2}\right)^{j} .
\end{aligned}
$$

According to our assumption, the only sources of the integrand singularities in the region $\operatorname{Re} j>-\frac{1}{2}$ are the zeros of the functions $\sin (\pi j)$ and $m_{ \pm}^{2}(j)-t$. Hence, deforming the contour $C$ to the axis $\operatorname{Re} j=-\frac{1}{2}$ (the behavior of $h_{ \pm}\left(t, j, m_{ \pm}^{2}(j)\right)$ at $j \rightarrow \infty$ and $\operatorname{Re} j>-\frac{1}{2}$ should allow such a deformation), we obtain

$$
\begin{aligned}
\delta_{ \pm}(s, t)= & \frac{1}{2 i} \int_{-\frac{1}{2}-i \infty}^{-\frac{1}{2}+i \infty} \frac{\mathrm{d} j}{\sin (\pi j)} \sum_{m_{ \pm}}\left(\mp 1-e^{-i \pi j}\right) \\
& \times \frac{h_{ \pm}\left(t, j, m_{ \pm}^{2}(j)\right)}{m_{ \pm}^{2}(j)-t}\left(\frac{s}{2}\right)^{j}+\sum_{n} \frac{\mp 1-e^{-i \pi \alpha_{n}^{ \pm}(t)}}{\sin \left(\pi \alpha_{n}^{ \pm}(t)\right)} \\
& \times \frac{\pi \mathrm{d} \alpha_{n}^{ \pm}(t)}{\mathrm{d} t} h_{ \pm}\left(t, \alpha_{n}^{ \pm}(t), t\right)\left(\frac{s}{2}\right)^{\alpha_{n}^{ \pm}(t)},
\end{aligned}
$$

where the functions $\alpha_{n}^{ \pm}(t)$ are the roots of the equations $m_{ \pm}^{2}(j)-t=0$ and, thus, they correspond to the eikonal poles in the complex $j$-plane. These poles are called Regge poles, and the functions $\alpha_{n}^{ \pm}(t)$ are called Regge trajectories.

At high enough values of $s$ the background integral contribution is negligible. As the functions $h_{ \pm}$can be factorized into two factors related to each of the interacting hadrons, so we arrive at the following expression for the eikonal:

$$
\begin{aligned}
\delta_{ \pm}(s, t)= & \sum_{n} \xi_{ \pm}\left(\alpha_{n}^{ \pm}(t)\right) g_{n}^{(1) \pm}(t) g_{n}^{(2) \pm}(t) \\
& \times \frac{\pi \mathrm{d} \alpha_{n}^{ \pm}}{\mathrm{d} t}\left(\frac{s}{2 s_{0}}\right)^{\alpha_{n}^{ \pm}(t)},
\end{aligned}
$$

where $s_{0}$ is some scale determined a priori (for example, $\left.s_{0}=1 \mathrm{GeV}^{2}\right)$ and related directly to the factors $g_{n}^{(i)}(t)$, which should be interpreted as the effective couplings of reggeons to the colliding particles. $\xi_{ \pm}(\alpha)$ are the so-called reggeon signature factors: $\xi_{+}(\alpha)=i+\operatorname{tg} \frac{\pi(\alpha-1)}{2}$ and $\xi_{-}(\alpha)=i-$ $\operatorname{ctg} \frac{\pi(\alpha-1)}{2}$.

The last formula (which is valid, as well, for inelastic scattering $2 \rightarrow 2$ and for reactions with off-shell particles), 
together with the eikonal representation (1) of the scattering amplitude, is the essence of the Regge-eikonal approach [2].

\section{References}

1. A.A. Godizov, Phys. Lett. B 735, 57 (2014)

2. P.D.B. Collins, An Introduction to Regge Theory and High Energy Physics (Cambridge University Press, Cambridge, 1977)

3. P.D.B. Collins, P.J. Kearney, Z. Phys. C 22, 277 (1984)

4. H. Cheng, T.T. Wu, Phys. Rev. Lett. 22, 666 (1969)

5. J.R. Cudell, A. Lengyel, E. Martynov. http://www.theo.phys.ulg. ac.be/ cudell/data. Accessed 19 June 2012

6. UA1 Collaboration, G. Arnison et al., Phys. Lett. B 128, 336 (1983)

7. UA4 Collaboration, M. Bozzo et al., Phys. Lett. B 147, 385 (1984)

8. UA4 Collaboration, M. Bozzo et al., Phys. Lett. B 155, 197 (1985)

9. UA4 Collaboration, D. Bernard et al., Phys. Lett. B 198, 583 (1987)
10. UA4 Collaboration, D. Bernard et al., Phys. Lett. B 171, 142 (1986)

11. E-710 Collaboration, N. Amos et al., Phys. Lett. B 247, 127 (1990)

12. The TOTEM Collaboration, Europhys. Lett. 101, 21002 (2013)

13. ATLAS Collaboration, Nucl. Phys. B 889, 486 (2014)

14. Particle Data Group. http://pdg.lbl.gov/2014/hadronic-xsections/ hadron.html. Accessed 22 Mar 2015

15. U. Amaldi, K.R. Schubert, Nucl. Phys. B 166, 301 (1980)

16. D0 Collaboration, Phys. Rev. D 86, 012009 (2012)

17. CDF Collaboration, F. Abe et al., Phys. Rev. D 50, 5518 (1994)

18. J. Kwiecinski, Phys. Rev. D 26, 3293 (1982)

19. R. Kirschner, Z. Phys. C 67, 459 (1995)

20. A.A. Godizov, V.A. Petrov, JHEP 0707, 083 (2007)

21. L. Van Hove, Phys. Lett. B 24, 183 (1967)

22. F. Carlson, Dissertation, Uppsala, Sweden, 1914

23. G.N. Watson, Proc. R. Soc. 95, 83 (1918)

24. A. Zommerfeld, Partial Differential Equations in Physics (Academic Press, New York, 1949) 